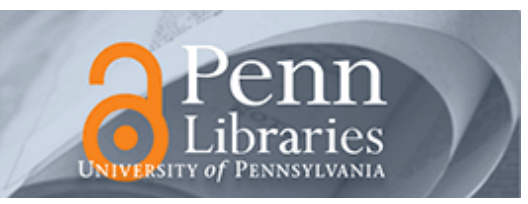

University of Pennsylvania

ScholarlyCommons

$11-14-2008$

\title{
Monte Carlo Analysis of Stress-Directed Phase Segregation in Binary Thin Film Alloys Under Nonisothermal Annealing
}

\author{
Alex M. Nieves \\ University of Pennsylvania, nievesam@seas.upenn.edu \\ Vaclav Vitek \\ University of Pennsylvania, vitek@seas.upenn.edu \\ Talid Sinno \\ University of Pennsylvania, talid@seas.upenn.edu
}

Follow this and additional works at: https://repository.upenn.edu/cbe_papers

Part of the Chemical Engineering Commons

\section{Recommended Citation}

Nieves, A. M., Vitek, V., \& Sinno, T. (2008). Monte Carlo Analysis of Stress-Directed Phase Segregation in Binary Thin Film Alloys Under Nonisothermal Annealing. Retrieved from https://repository.upenn.edu/ cbe_papers/142

Suggested Citation:

Nieves, A.M., V. Vitek, and T. Sinno. (2008). "Monte Carlo analysis of stress-directed phase segregation in binary thin film alloys under nonisothermal annealing." Applied Physics Letters. 93, 19194.

(C) 2008 American Institute of Physics. This article may be downloaded for personal use only. Any other use requires prior permission of the author and the American Institute of Physics. The following article appeared in Applied Physics Letters and may be found at http://dx.doi.org/10.1063/1.3027064.

This paper is posted at ScholarlyCommons. https://repository.upenn.edu/cbe_papers/142

For more information, please contact repository@pobox.upenn.edu. 


\title{
Monte Carlo Analysis of Stress-Directed Phase Segregation in Binary Thin Film Alloys Under Nonisothermal Annealing
}

\author{
Abstract \\ The use of patterned stress fields to direct phase separation in thin film alloys is investigated \\ computationally with Monte Carlo simulations in which atomic interactions are represented by a Lennard- \\ Jones potential.We show that careful design of annealing schedules based on consideration of the \\ system phase diagram can lead to vastly enhanced patterning kinetics. In particular, by avoiding the low \\ temperature formation of highly stable nuclei within the entire system, the kinetics of patterning are \\ accelerated by rapid monomer diffusion, rather than classical Ostwald ripening in which small \\ precipitates must dissolve to feed larger ones. \\ Disciplines \\ Chemical Engineering | Engineering

\section{Comments} \\ Suggested Citation: \\ Nieves, A.M., V. Vitek, and T. Sinno. (2008). "Monte Carlo analysis of stress-directed phase segregation in \\ binary thin film alloys under nonisothermal annealing." Applied Physics Letters. 93, 19194. \\ (C) 2008 American Institute of Physics. This article may be downloaded for personal use only. Any other \\ use requires prior permission of the author and the American Institute of Physics. The following article \\ appeared in Applied Physics Letters and may be found at http://dx.doi.org/10.1063/1.3027064.
}




\title{
Monte Carlo analysis of stress-directed phase segregation in binary thin film alloys under nonisothermal annealing
}

\author{
Alex M. Nieves, ${ }^{1}$ V. Vitek, ${ }^{2}$ and T. Sinno ${ }^{1, a)}$ \\ ${ }^{1}$ Department of Chemical and Biomolecular Engineering, University of Pennsylvania, Philadelphia, \\ Pennsylvania 19104, USA \\ ${ }^{2}$ Department of Materials Science and Engineering, University of Pennsylvania, Philadelphia, \\ Pennsylvania 19104, USA
}

(Received 23 September 2008; accepted 25 October 2008; published online 14 November 2008)

\begin{abstract}
The use of patterned stress fields to direct phase separation in thin film alloys is investigated computationally with Monte Carlo simulations in which atomic interactions are represented by a Lennard-Jones potential. We show that careful design of annealing schedules based on consideration of the system phase diagram can lead to vastly enhanced patterning kinetics. In particular, by avoiding the low temperature formation of highly stable nuclei within the entire system, the kinetics of patterning are accelerated by rapid monomer diffusion, rather than classical Ostwald ripening in which small precipitates must dissolve to feed larger ones. (C) 2008 American Institute of Physics.

[DOI: 10.1063/1.3027064]
\end{abstract}

Several experimental and computational studies have demonstrated that applied stress fields can direct the formation of precipitation or spinodal decomposition patterns at surfaces $^{1-5}$ or in the bulk ${ }^{6-9}$ of binary systems. Examples include atomic rearrangement on vicinal $\mathrm{Si}(100)$ due to substrate bending, ${ }^{10}$ ordering of arsenic precipitation within GaAs heterostructures with fabricated stressors, ${ }^{6}$ and directed Ge island growth on prepatterned $\mathrm{Si}(001)$ surfaces. ${ }^{4}$ In this letter, we focus on a controlled application of this general concept. Specifically, using Metropolis Monte Carlo (MMC) with atomic interactions described by a LennardJones (LJ) potential, we investigate how to control the location of precipitates formed in a thin film of a supersaturated binary solid solution by the application of indenters at the surface of the film. It is envisaged that the compression in the regions below the indenters would favor the presence of smaller atomic species in a mismatched binary system.

The MMC simulation domain employed here is shown in Fig. 1(a). The length-to-height ratio of the film is 128:12 and the domain is comprised of 49152 atoms of type $A$ or $B$ arranged in an fcc lattice. $B$ atoms are defined as the minority species that are smaller than $A$ atoms and form precipitates that are strained in hydrostatic tension within the $A$ rich matrix. Periodic boundary conditions are applied in the $x$ and $y$ directions. Both surfaces normal to $z$ are subject to a fixed displacement in the $z$ direction that corresponds, within the period, to two indenters with cylindrical symmetry. Note that in-plane atomic relaxation is still permitted on the surfaces. In all simulations the initial configuration consisted of a random solid solution containing $70 \% \mathrm{~A}$ atoms and $30 \% \mathrm{~B}$ atoms. An example of the hydrostatic stress field that results from the application of two cylindrical indenters on the top and bottom surfaces of the thin film is shown in Fig. 1(b).

Within the MMC, the system shown in Fig. 1 was evolved via atomic identity exchanges between randomly chosen nearest-neighboring particles. An identity switch attempt was accepted or rejected according to the usual Metropolis criterion at the prescribed temperature. Note that

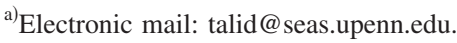

atomic identity exchanges between atoms of the same type do not alter the system configuration in any way but are included in the total count of switch attempts that is used to monitor the process evolution. In real crystalline systems atomic diffusion is mediated by point defects (e.g., vacancies in metals ${ }^{11}$ ) that are not explicitly considered here. However, under the assumption of a uniform (i.e., stress and composition independent) point defect distribution and diffusivity, the total number of random neighboring atomic identity exchanges is further assumed to provide a qualitative measure of the total point defect-mediated natural process within the system. Atomic relaxations in the neighborhood of identity switches (between unlike particles) are captured by periodically relaxing the entire system every $5 \times 10^{6}$ switch attempts using a conjugate gradient energy minimization technique. More frequent relaxations were found to increase the computational cost of the simulation without notable effect on the predicted dynamic evolution. All simulations were performed within the $N-P-T$ ensemble at zero total hydrostatic pressure, which was controlled by isotropic volume expansion/compression MMC moves. ${ }^{12}$

The $A-A, A-B$, and $B-B$ atomic interactions were described by a binary $\mathrm{LJ}$ pair potential

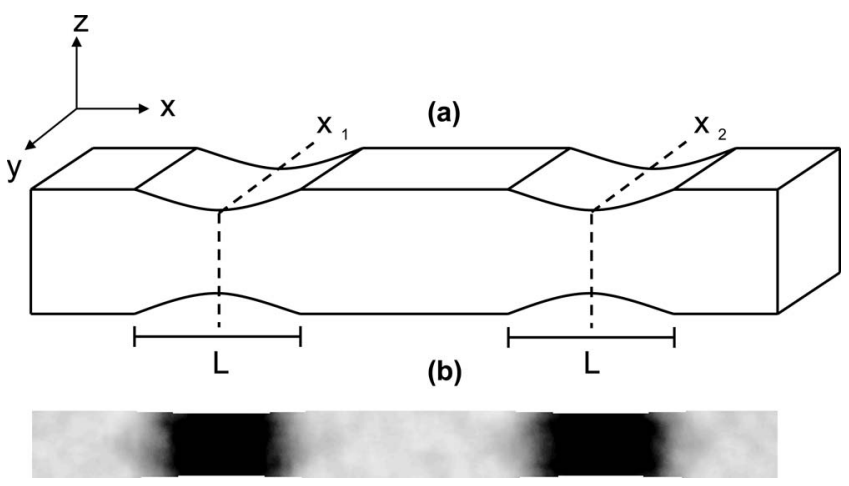

FIG. 1. (a) Schematic representation of the geometry of the simulation domain showing cylindrical intenders applied at top and bottom surfaces. (b) Instantaneous hydrostatic stress field within an $x z$-cross section of the simulation domain. Dark shade represents compression while light shade denotes tension. 
TABLE I. Length and energy scale parameters for the LJ potential.

\begin{tabular}{lccc}
\hline \hline & $\begin{array}{c}A-A \\
\text { interaction }\end{array}$ & $\begin{array}{c}A-B \\
\text { interaction }\end{array}$ & $\begin{array}{c}B-B \\
\text { interaction }\end{array}$ \\
\hline Length parameter $\sigma(\AA)$ & 3.405000 & 3.353925 & 3.302850 \\
Energy parameter $\varepsilon(\mathrm{eV})$ & 0.0103230 & 0.0092907 & 0.0103230 \\
\hline
\end{tabular}

$$
E\left(r_{i j}\right)=4 \varepsilon_{A i A j}\left[\left(\frac{\sigma_{A i A j}}{r_{i j}}\right)^{12}-\left(\frac{\sigma_{A i A j}}{r_{i j}}\right)^{6}\right],
$$

where $A_{i}$ is the species type of atom $i .^{13}$ The length parameter $\sigma$ for the $A-A$ interaction has been chosen to be larger than for the $B-B$ interaction in order to simulate a misfit between the $A$ and $B$ phases that couples with the externally applied stress field. The length parameter for $A-B$ interactions was taken as the arithmetic average of the $A-A$ and $B-B$ values. The energy parameters $\varepsilon_{A A}$ and $\varepsilon_{B B}$ were chosen to be the same, while $\varepsilon_{A B}$ was lowered by $10 \%$ to drive phase segregation. The LJ parameters used in this study are summarized in Table I and correspond to a misfit strain of 3\% between the larger $A$ atoms and smaller $B$ atoms. The cutoff distance for all atomic interactions $\left(r_{c}\right)$ was set to $7.5 \AA$ and a third-order polynomial tail was employed to zero the energy and its derivative at $r_{c}$. All temperatures reported in the following discussion are scaled by the maximum solvus temperature, i.e., $T^{*} \equiv T / T_{C}$, where $T_{C}$ was calculated to be $85 \mathrm{~K}$ (or $0.97 T_{m}$ ) for the potentials used [see Fig. 2(b) and accompanying discussion for details].

Constant temperature simulations were performed at $T^{*}$ $=0.35, T^{*}=0.59$, and $T^{*}=0.82$. The extent of patterning in

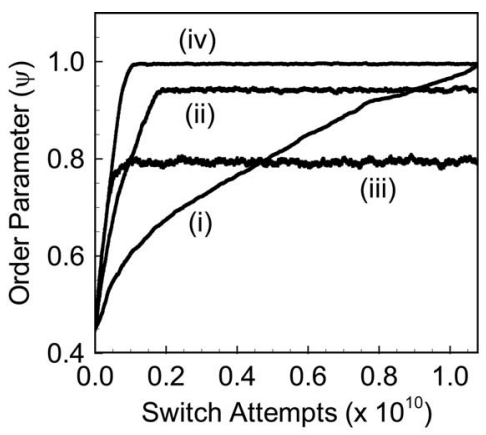

(a)

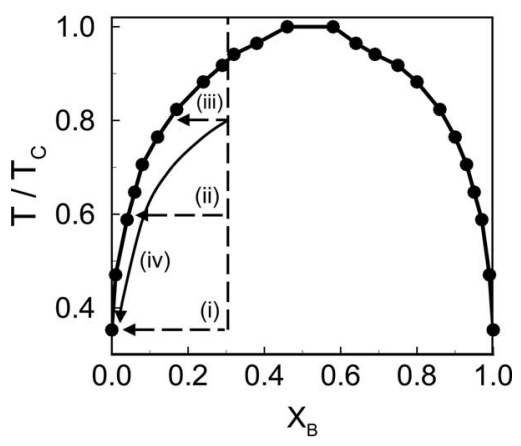

(b)

FIG. 2. (a) Patterning extent $\psi$ as a function of total identity switch attempts: (i) $T^{*}=0.35$, (ii) $T^{*}=0.59$, (iii) $T^{*}=0.82$, and (iv) nonisothermal anneal with $0.35 \leq T^{*} \leq 0.82$. (b) Phase diagram for binary LJ alloy with parameters defined in Table I. Dashed (isothermal) and solid (variable temperature) path lines represent corresponding compositional evolution pathway of the matrix phase away from the indenters. the system was measured by an order parameter $\psi$ equal to the fraction of $B$ atoms located in the regions below the indenters, defined by $-L / 2 \leq x-x_{1} \leq L / 2$ and $-L / 2 \leq x-x_{2}$ $\leq L / 2$ (see Fig. 1). The evolution of $\psi$ as a function of identity switch attempts for the three constant-temperature anneals is shown in Fig. 2(a). The initial value of $\psi \sim 0.45$ corresponds to homogeneously distributed $B$ species throughout the simulation block. As the temperature is increased the maximum (equilibrium) value of $\psi$ attained decreases because the matrix and precipitate phases become increasingly compositionally mixed due to configurational entropy. Only at (and below) $T^{*}=0.35$ does the system fully pattern after $1 \times 10^{10}$ switch attempts.

The equilibrium compositions of the matrix and precipitate phases as a function of temperature were computed with independent semigrand canonical MMC simulations, ${ }^{14}$ see Fig. 2(b). The computed solubility curve shows that at temperatures above $T^{*} \sim 0.4$, both phases contain increasing amounts of the minority species at equilibrium and it is therefore not possible to achieve complete patterning, i.e., $\psi=1$. The three dashed lines, which represent the three constant-temperature anneals denoted by labels (i)-(iii), show the final equilibrium composition of the matrix phase when an initially homogeneous solid solution at high temperature containing $30 \% \mathrm{~B}$ atoms is quenched (vertical segments) and annealed at constant temperature (horizontal segments). Hence, at constant temperature, a compromise must be achieved between the speed of the evolution and the achievable equilibrium extent of patterning.

Next, a nonisothermal simulation was performed in which the starting temperature was $T^{*}=0.82$. The system was allowed to evolve at this temperature for about $2.5 \times 10^{8}$ switch attempts, after which the temperature was decreased linearly at a rate of 0.1 scaled degrees per $2 \times 10^{8}$ switch attempts until it reached $T^{*}=0.35$ at $1.2 \times 10^{9}$ switch attempts. The remainder of the simulation was performed at $T^{*}=0.35$. The evolution of $\psi$ for this run (iv) is shown in Fig. 2(a) along with the constant-temperature curves, (i)-(iii). Clearly, the rate of patterning in this simulation is dramatically increased relative to the constant-temperature runs, while also achieving complete patterning. This large acceleration can be understood by considering the path denoted by the solid line in Fig. 2(b). During the initial high-temperature phase, which is only slightly below the solvus, the driving force for nucleation of the $B$-rich phase is low. Moreover, as shown in Fig. 2(b), any precipitate that is formed at this high temperature is only slightly enriched in $B$ relative to the surrounding solid solution and therefore weakly bound. Meanwhile, as the stress field continuously drives $B$ atoms toward the indenters, the system temperature can be lowered without causing additional precipitation away from the indenters, as shown schematically by the constant proximity of the solid line path in Fig. 2(b) to the solvus throughout the anneal. The result is that the transport of $B$ to the indenters is achieved almost entirely by monomer diffusion or by dissolution of weakly bound precipitates formed at high temperature.

The compositional evolution within the two types of processes is contrasted explicitly in Fig. 3, which shows twodimensional slices of the simulation block as a function of identity switch attempts for the $T^{*}=0.35$ anneal [process (i), left side] and the nonisothermal anneal [process (iv), right side]. In the isothermal case, immediate (low temperature) formation of precipitates that are almost pure $B$ depletes the 


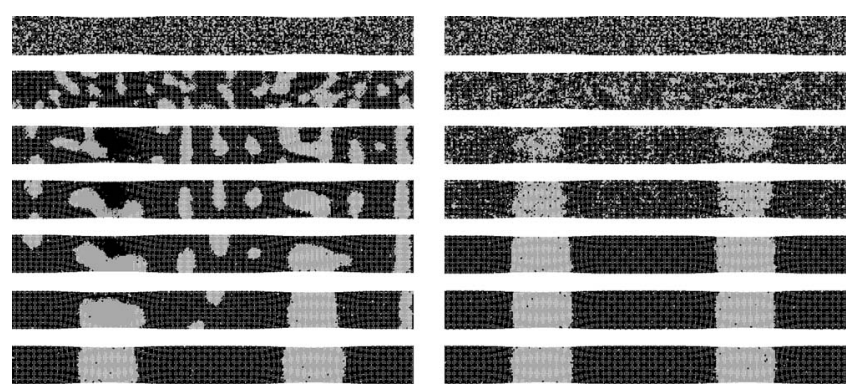

FIG. 3. Morphological evolution as a function of total switch attempts for two annealing processes of an initially homogenous solid solution. $A$ atoms are black and $B$ atoms light gray. Left side: isothermal anneal at $T^{*}=0.35$. Right side: variable temperature anneal $0.35 \leq T^{*} \leq 0.82$. From top to bottom, the images on each row are taken at: $0\left(T^{*}=0.82\right), 2.5 \times 10^{7}\left(T^{*}\right.$ $=0.82), 2.5 \times 10^{8} \quad\left(T^{*}=0.82\right), 5.5 \times 10^{8} \quad\left(T^{*}=0.55\right), 1.2 \times 10^{9} \quad\left(T^{*}=0.35\right)$, $5.0 \times 10^{9}\left(T^{*}=0.35\right)$, and $1.1 \times 10^{10}\left(T^{*}=0.35\right)$ switch attempts, respectively. The quoted temperatures correspond to the variable temperature anneal.

surrounding matrix throughout the simulation domain. The nonisothermal process, however, accomplishes two objectives during the early stages of the anneal. First, it slows the formation of large $B$ precipitates everywhere and second, as discussed earlier, it ensures that the precipitates that do form are only slightly enriched in $B$ and thus weakly bound; in fact the precipitates in the second panel on the right side of Fig. 3 are barely discernable throughout the domain. Both effects make easier, relative to the isothermal case, the stressmediated net transport of $B$ monomers to the indenters. As a result, complete $B$ patterning is achieved in less than $10 \%$ of the switch attempts needed for complete patterning in the isothermal case at $T^{*}=0.35$.

In summary, we have demonstrated using quasistatic MMC simulations that the dynamics of precipitate patterning in an alloy, driven by an externally imposed field such as stress, can be greatly enhanced with appropriately designed thermal anneals. In particular, variable temperature annealing allows for the tuning of nucleation/precipitation driving forces relative to stress-mediated segregation, which in turn enables patterning to proceed by rapid monomer diffusion and coarsening of weakly bound precipitates that were formed at high temperatures. We note that the present results are not qualitatively affected by the particular choice of the surface energy and misfit parameters. Increasing (decreasing) the surface energy penalty and/or the misfit between the two species reduces (increases) their miscibility and increases (decreases) the solvus temperature. Very large misfits $(>6-7 \%)$, however, also lead to plastic deformation during phase segregation, which is not considered here and will be addressed in future work. Further studies will be aimed at developing quantitative guidelines that can be used to select realistic systems that are optimally suitable for field-directed assembly.

We gratefully acknowledge financial support from the National Science Foundation through NIRT Award No. CTS-0404259. We also acknowledge valuable discussions with Yucun Lou and John L. Bassani.

${ }^{1}$ T. R. Mattsson and H. Metiu, Appl. Phys. Lett. 75, 926 (1999).

${ }^{2}$ R. F. Sabiryanov, M. I. Larsson, K. J. Cho, W. D. Nix, and B. M. Clemens, Phys. Rev. B 67, 125412 (2003).

${ }^{3}$ W. Lu and Z. Suo, J. Mech. Phys. Solids 49, 1937 (2001).

${ }^{4}$ Z. Zhong, P. Chen, Z. Jiang, and G. Bauer, Appl. Phys. Lett. 93, 043106 (2008).

${ }^{5}$ A. Ohtake and N. Koguchi, Appl. Phys. Lett. 89, 083108 (2006).

${ }^{6}$ C.-Y. Hung, A. F. Marshall, D.-K. Kim, W. D. Nix, J. S. Harris, Jr., and R. A. Kiehl, J. Nanopart. Res. 1, 329 (1999).

${ }^{7}$ Y. Lou and J. L. Bassani, J. Mech. Phys. Solids 56, 3507 (2008).

${ }^{8}$ D. Y. Li and L. Q. Chen, Acta Mater. 46, 2573 (1998).

${ }^{9}$ A. W. Zhu and E. A. Starke, Jr., Acta Mater. 49, 2285 (2001).

${ }^{10}$ C. S. Chang and T. T. Tsong, Phys. Rev. Lett. 77, 2021 (1996).

${ }^{11}$ S. Balaji, T. A. Abinandanan, K. G. Ayappa, and N. Chandrasekhar, Philos. Mag. A 80, 301 (2000).

${ }^{12}$ M. P. Allen and D. J. Tildesley, Computer Simulation of Liquids (Oxford University Press, New York, 1989), p. 123.

${ }^{13}$ J. P. K. Doye and L. Meyer, Phys. Rev. Lett. 95, 063401 (2005).

${ }^{14}$ D. Frenkel and B. Smit, Understanding Molecular Simulation: From Algorithms to Applications (Academic Press, New York, 2002), p. 225. 